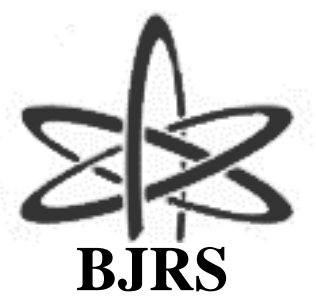
BRAZILIAN JOURNAL
$\mathrm{OF}$
RADIATION SCIENCES
06-02-B (2018) 01-17

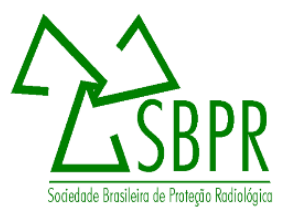

\title{
10 Recomendações para prevenção de acidentes radiológicos em gamagrafia industrial
}

\author{
L. S. Souza ${ }^{a}$; F. C. A. Da Silva ${ }^{\mathrm{b}}$ \\ ${ }^{a}$ Pós-Graduação Lato Sensu em Proteção Radiológica e Segurança de Fontes Radioativas, \\ ${ }^{b}$ Instituto de Radioproteção e Dosimetria/CNEN, 22783-127, Rio de Janeiro-RJ, Brasil \\ dasilva@ird.gov.br
}

\begin{abstract}
RESUMO
A gamagrafia industrial desempenha um papel importante no controle de qualidade de diversos materiais e peças. É classificada pela AIEA como Categoria 2, devido ao seu risco radiológico provocado pelo uso de fontes radioativas de alta atividade. Esse risco está baseado nas consequências danosas geradas à saúde humana, descrito em alguns acidentes ocorridos no mundo, devido a falhas cometidas. Em 2012, foi realizado pela DIAPI/CNEN, o "Workshop Nacional sobre Prevenção de Acidente em Gamagrafia Industrial", com o objetivo de disseminar conhecimento sobre os acidentes radiológicos. Na ocasião, o IRD/CNEN realizou uma pesquisa com os 75 participantes usando um formulário com 22 recomendações para prevenir acidentes radiológicos, objetivando selecionar as 10 mais votadas. Um estudo estatístico, usando o método "Distribuição de Frequências", foi realizado para definir 10 recomendações. Os resultados percentuais e de votos foram obtidos por categoria dos participantes e foram definidas as 10 recomendações mais importantes para prevenir acidentes radiológicos. A recomendação que ficou em $1^{\circ}$ lugar foi "Use sempre um monitor individual com alarme durante todo o trabalho".
\end{abstract}

Palavras-chave: gamagrafia Industrial; risco radiológico; prevenção de acidentes radiológicos.

ISSN: 2319-0612

Accepted 2018-08-08 


\begin{abstract}
Industrial gamma radiography plays an important role in quality control of various materials and components. IAEA classifies it as Category 2, due to its radiological risk caused by high radioactive sources activities. This risk is based on the harmful effects on human health, described in some accidents, due to operation failures. In 2012, a "National Workshop on Prevention of Industrial Gamma Radiography Accidents", was held by DIAPI/CNEN to disseminate information about radiological accidents. At the time, IRD/CNEN carried out a survey with 75 participants using a form with 22 recommendations to prevent radiological accidents, in order to select the top 10 voted. A statistical study, using the "Frequency Distribution" method, was performed to define the top 10 recommendations. The results of percentage and vote were obtained for each participant category and the top 10 recommendations were defined to prevent radiological accidents. The first and most voted recommendation was "Always use an individual alarm monitor during entire work".
\end{abstract}

Keywords: Industrial gamma radiography; radiological risk; radiological accidents prevention.

\title{
1. INTRODUÇÃO
}

A gamagrafia industrial é uma das técnicas mais difundidas entre as aplicações nucleares na indústria, pois não utiliza energia elétrica e possui modelos portáteis de fácil locomoção. Desempenha um papel importante na inspeção de componentes, para o controle da qualidade de soldas, materiais, peças e estruturas na indústria naval, petrolífera e siderúrgica.

A prática de gamagrafia industrial requer equipamentos que irão auxiliar nos ensaios e desempenho das funções de proteção radiológica, a fim de que o trabalhador não se exponha a altas doses de radiação. Basicamente para um ensaio é necessário ter um irradiador de gamagrafia, contendo urânio exaurido para a blindagem da fonte radioativa; um tubo guia, que acoplado ao irradiador conduzirá a fonte; um cabo de comando com controle mecânico, para a movimentação da fonte; e tripé que, caso necessário, irá fixar o terminal de exposição quando a fonte for exposta sobre o material.

Os equipamentos mínimos de proteção radiológica necessários para o ensaio são, basicamente, o monitor individual de dose, monitor individual de alarme o e monitor portátil de radiação de área. 
A gamagrafia industrial utiliza fontes radioativas, emissoras de raios gama, de ${ }^{192} \mathrm{Ir},{ }^{75} \mathrm{Se} \mathrm{e}$

${ }^{60}$ Co. Possui um elevado nível de risco radiológico devido as altas atividades das fontes (até 3.7 $\mathrm{TBq}$ ), da probabilidade de consequências danosas que podem ocorrer à saúde humana quando da ocorrência de falhas durante o uso, e ao elevado número de acidentes radiológicos com lesões [1]. É classificada pela IAEA, devido as atividades, como Categoria 2 de "muito perigosa" [2]. Alguns acidentes radiológicos já ocorreram na América Latina e no Brasil, podendo ser citado: Acidente Radiológico em Nova Aldea no Chile [3]; o Acidente Radiológico em Yanango no Peru [4]; e o Acidente Radiológico em São Paulo no Brasil [1].

Com o objetivo de disseminar as lições aprendidas, para prevenir acidentes radiológicos com fontes de gamagrafia industrial em todo país, foi realizado, pela DIAPI/CNEN/RJ, o "Workshop Nacional sobre Prevenção de Acidentes em Gamagrafia Industrial”, no qual o IRD/CNEN realizou uma pesquisa com os participantes usando um formulário com 22 recomendações para prevenir acidentes radiológicos. Este trabalho apresenta estatisticamente as 10 recomendações mais votadas.

\section{MATERIAIS E MÉTODOS}

A coleta de dados foi realizada durante o Workshop através de um formulário, elaborado pelo IRD/CNEN, com 22 recomendações para prevenir acidentes radiológicos, previamente elaborado baseado nas lições aprendidas de acidentes radiológicos e nas recomendações nacionais e internacionais. O referido questionário foi distribuído para os 75 participantes divididos em quatro categorias: Geral (75 participantes), SPR (29 participantes), Trabalhadores da área (25 participantes) e Outros (CNEN e não identificados 21 participantes). Foi solicitado que escolhessem somente 10 recomendações e classificá-las na importância de 1 a 10, sendo o número um (1) o mais importante (figura 1). 
Figura 1: Formulário com as 22 recomendações para prevenção de acidentes em gamagrafia industrial

\begin{tabular}{|c|c|c|c|}
\hline \multicolumn{4}{|c|}{$\begin{array}{c}\text { WORKSHOP NACIONAL SOBRE PREVENÇ̃̃O DE ACIDENTES EM GAMAGRAFIA INDUSTRIAL } \\
\text { CNEN 27-28/Março/2012 }\end{array}$} \\
\hline \multicolumn{4}{|c|}{ Levantamento de dados proposto pela Área de Indústria do IRD/CNEN } \\
\hline \multicolumn{4}{|c|}{$\begin{array}{l}\text { 10 RECOMENDAÇÕES IMPORTANTES PARA O OPERADOR DE GAMAGRAFIA INDUSTRIAL } \\
\text { EVITAR ACIDENTES RADIOLÓGICOS }\end{array}$} \\
\hline \multicolumn{3}{|c|}{ Participante: } & \begin{tabular}{l|l} 
& e-mail: \\
\end{tabular} \\
\hline \# & $\begin{array}{l}\text { Escolha } \\
\text { somente } \\
10\end{array}$ & $\begin{array}{c}\text { Classifique as } \\
\text { escolhidas de } \\
1 \text { (a mais } \\
\text { importante) a } \\
10\end{array}$ & $\begin{array}{c}\text { Recomendações importantes para o OPERADOR de gamagrafia industrial } \\
\text { EVITAR acidentes radiológicos } \\
\text { (em ordem alfabética) }\end{array}$ \\
\hline 1. & & & $\begin{array}{l}\text { Avalie com antecedência o local da radiografia antes da realização do } \\
\text { trabalho. }\end{array}$ \\
\hline 2. & & & $\begin{array}{l}\text { Comunique ao Supervisor de Radioproteção as possíveis anormalidades na } \\
\text { operação e nos equipamentos. }\end{array}$ \\
\hline 3. & & & Conecte corretamente dos engates do cabo de comando e do porta-fonte. \\
\hline 4. & & & Conheça muito bem como operar o tipo de irradiador a ser usado. \\
\hline 5. & & & $\begin{array}{l}\text { Faça o teste com o gabarito "passa-não passa" no porta-fonte e no cabo de } \\
\text { comando. }\end{array}$ \\
\hline 6. & & & Guarde o irradiador em um local adequado e seguro. \\
\hline 7. & & & Inspecione o veículo antes do transporte do irradiador com fonte radioativa. \\
\hline 8. & & & $\begin{array}{l}\text { Inspecione os equipamentos de radiografia e acessórios para verificar as } \\
\text { boas condições de uso. }\end{array}$ \\
\hline 9. & & & Mantenha a área de exposição isolada e sinalizada. \\
\hline 10. & & & Mantenha o irradiador sempre vigiado. \\
\hline 11. & & & Monitore a área depois de recolher a fonte radioativa ao final dos trabalhos. \\
\hline 12. & & & $\begin{array}{l}\text { Monitore o irradiador, antes de iniciar os trabalhos, para verificar o nível de } \\
\text { radiação inicial. }\end{array}$ \\
\hline 13. & & & Monitore sempre o irradiador e o tubo-guia após recolher a fonte radioativa. \\
\hline 14. & & & Nunca toque na fonte radioativa do porta-fonte de gamagrafia. \\
\hline 15. & & & $\begin{array}{l}\text { Realize ações de emergência somente se tiver devidamente treinado, } \\
\text { usando equipamentos específicos e sob supervisão. }\end{array}$ \\
\hline 16. & & & Trabalhe somente com uma equipe de radiografia completa e qualificada. \\
\hline 17. & & & $\begin{array}{l}\text { Trabalhe somente se suas condições físicas e psicológicas estiverem dentro } \\
\text { da normalidade. }\end{array}$ \\
\hline 18. & & & Trave sempre o irradiador após o final de cada exposição. \\
\hline 19. & & & Use sempre o monitor de radiação ao entrar na área isolada de radiografia. \\
\hline 20. & & & Use sempre um monitor individual com alarme durante todo o trabalho. \\
\hline 21. & & & Verifique o funcionamento correto dos monitores de radiação em uso. \\
\hline 22. & & & Verifique visualmente a posição da fonte radioativa no irradiador. \\
\hline
\end{tabular}


Para a análise dos dados foi utilizado o método estatístico de "Distribuição de Frequências" [5], baseado em agrupar dados, de modo a fornecer a quantidade ou porcentagem dos dados organizados em grupos ou categorias, possibilitando assim uma classificação geral das 22 recomendações e das 10 mais votadas. A análise foi realizada nas seguintes etapas:

- 1a . Etapa: foram contabilizadas todas as 22 recomendações classificando-as para os 75 participantes e foram retiradas as 10 mais votadas.

- 2a . Etapa: foi feita uma separação dos participantes em quatro categorias: Geral (75 participantes), SPR (29 participantes), Trabalhadores da área (25 participantes) e Outros (CNEN e não identificados, 21 participantes), em seguida, foram contabilizadas as 22 recomendações de cada categoria de acordo com a quantidade de participantes, classificando as 10 mais votadas.

- $3^{\text {a }}$ Etapa: tomando por base as 10 recomendações mais votadas da categoria Geral, foi contabilizado o número de votos para cada uma em relação às três categorias (SPR, Trabalhadores da área e outros).

\section{RESULTADOS E DISCUSSÕES}

Os resultados a seguir, estão baseados de acordo com a metodologia usada para a contabilização do formulário com as 22 recomendações [6].

\subsection{Classificação Geral}

Na classificação da categoria "Geral” foi quantificado o resultado final dos 75 participantes nas 22 recomendações, destacando-se com 8\% dos votos a questão 20 (Use sempre um monitor individual com alarme durante todo o trabalho), se tornando $1^{\circ}$ lugar entre as 10 mais votadas com $77 \%$ (58 votos), conforme mostram as figuras 2,3 e 4 . 
Figura 2: Resultado da categoria Geral

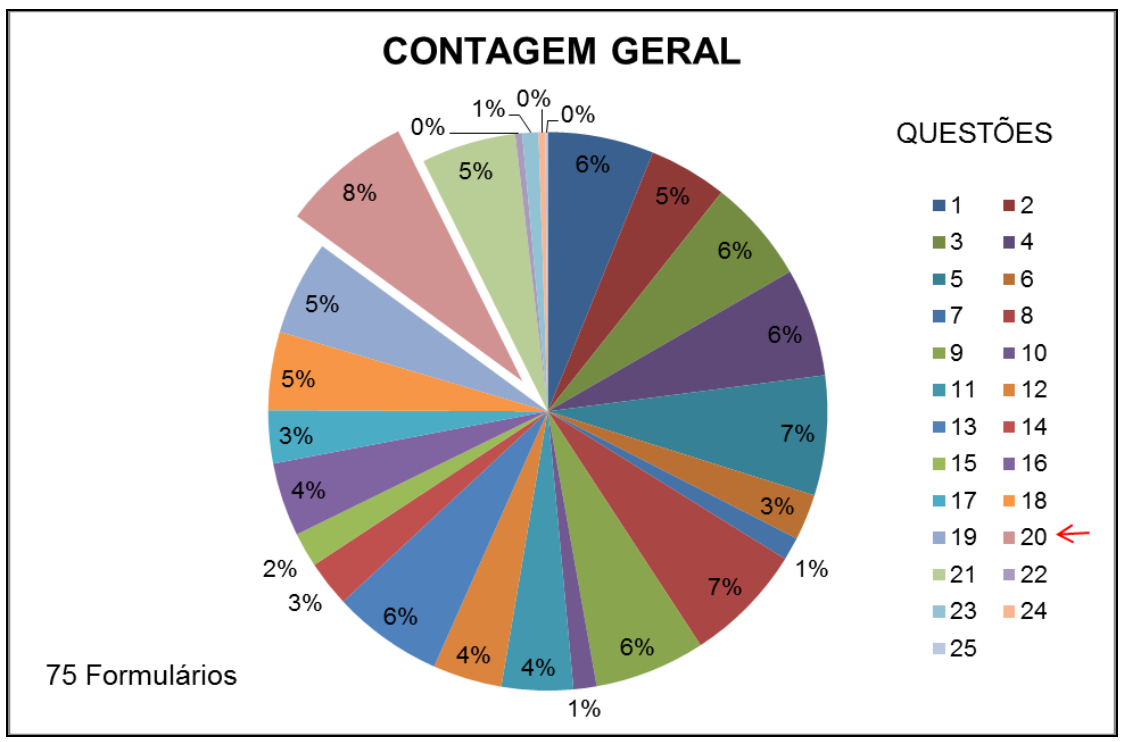

Fonte: SOUZA, 2015

Figura 3: Percentual das mais votadas na categoria Geral

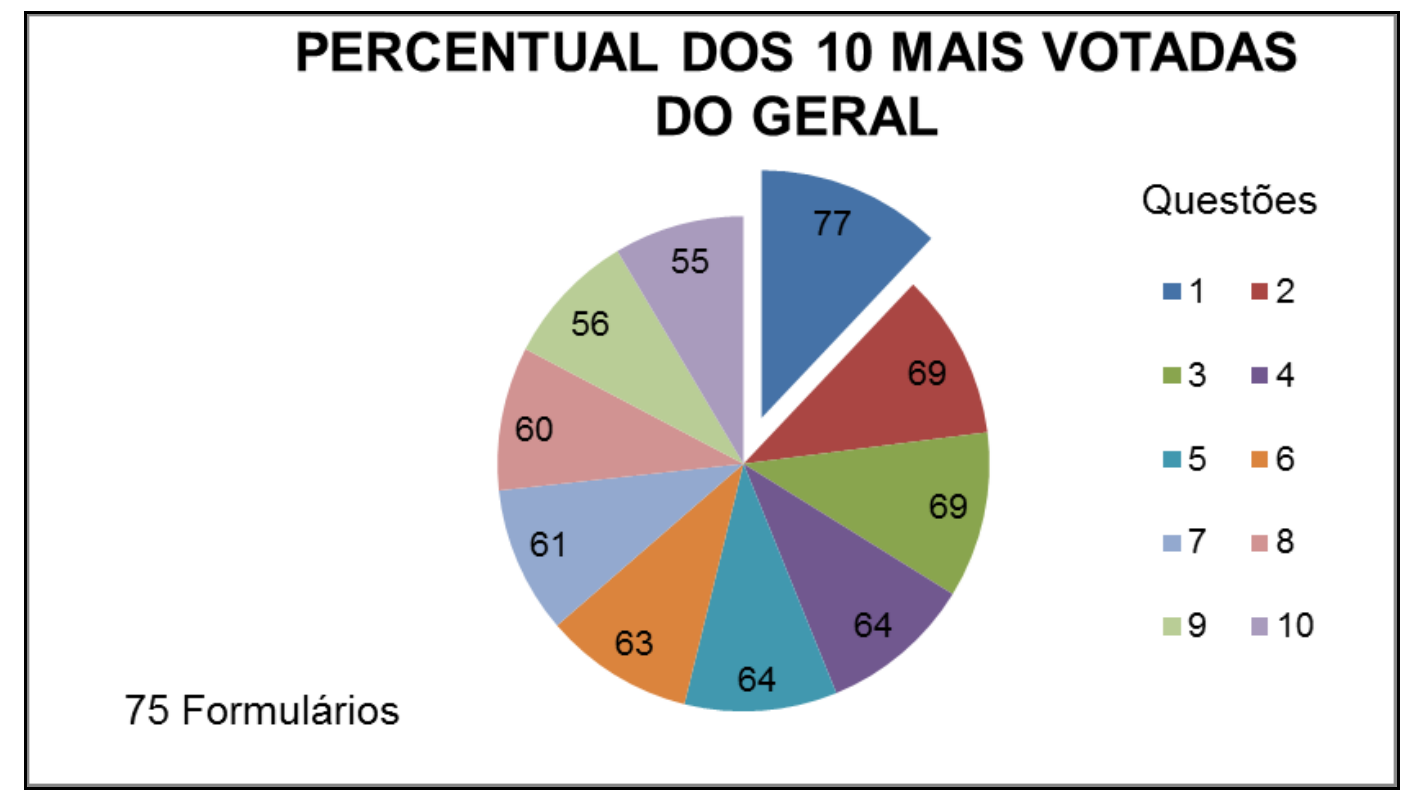

Fonte: SOUZA, 2015 
Figura 4: Quantidade do número de votos da categoria Geral

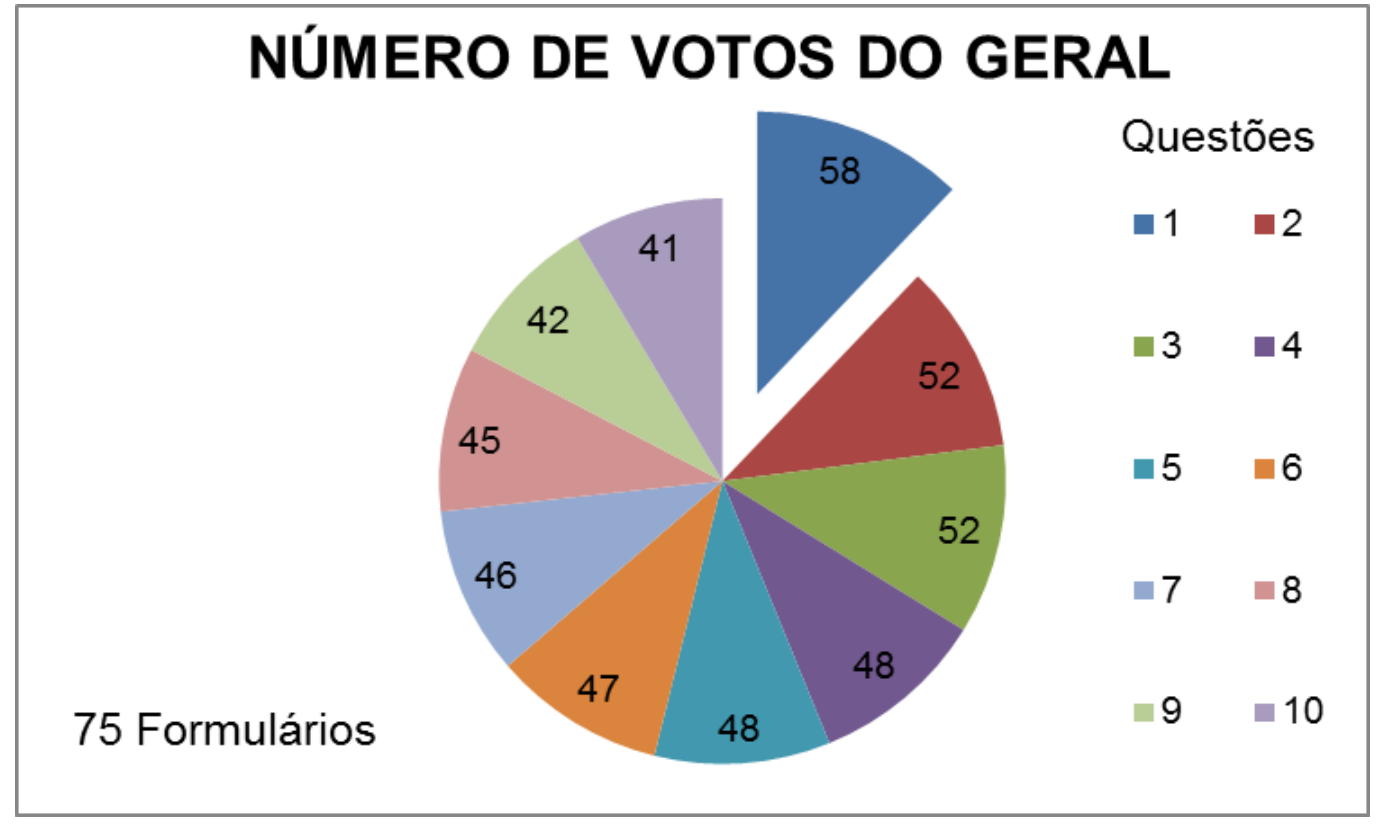

Fonte: SOUZA, 2015

E assim, as 10 recomendações mais votadas na categoria "Geral" foram:

$1^{\mathrm{a}}$. Use sempre um monitor individual com alarme durante todo o trabalho.

$2^{\mathrm{a}}$. Inspecione os equipamentos de radiografia e acessórios para verificar as boas condições de uso.

$3^{\text {a }}$. Faça o teste do gabarito "passa-não passa" no porta-fonte e no cabo de comando.

$4^{\mathrm{a}}$. Mantenha a área de exposição isolada e sinalizada.

5a . Monitore sempre o irradiador e o tubo-guia após recolher a fonte.

$6^{\mathrm{a}}$. Conheça muito bem como operar o tipo de irradiador a ser usado.

$7^{\mathrm{a}}$. Avalie com antecedência o local da radiografia antes da realização do trabalho.

$8^{a}$. Conecte corretamente os engates do cabo de comando e do porta-fonte.

$9^{a}$. Use sempre o monitor de radiação ao entrar na área isolada de radiografia.

$10^{\mathrm{a}}$. Verifique o funcionamento correto dos monitores de radiação em uso. 


\subsection{Classificação das Três Categorias (SPR, Trabalhadores da Área e Outros)}

Na classificação da categoria "SPR" foi quantificado o resultado dos 29 participantes nas 22 recomendações, destacando-se com $10 \%$ dos votos a questão 20 (Use sempre um monitor individual com alarme durante todo o trabalho), sendo o $1^{\circ}$ lugar entre as 10 mais votadas com 97\% (28 votos), sendo igual ao da categoria "Geral”, como mostram as figuras 5, 6 e 7.

Figura 5: Resultado geral da categoria SPR

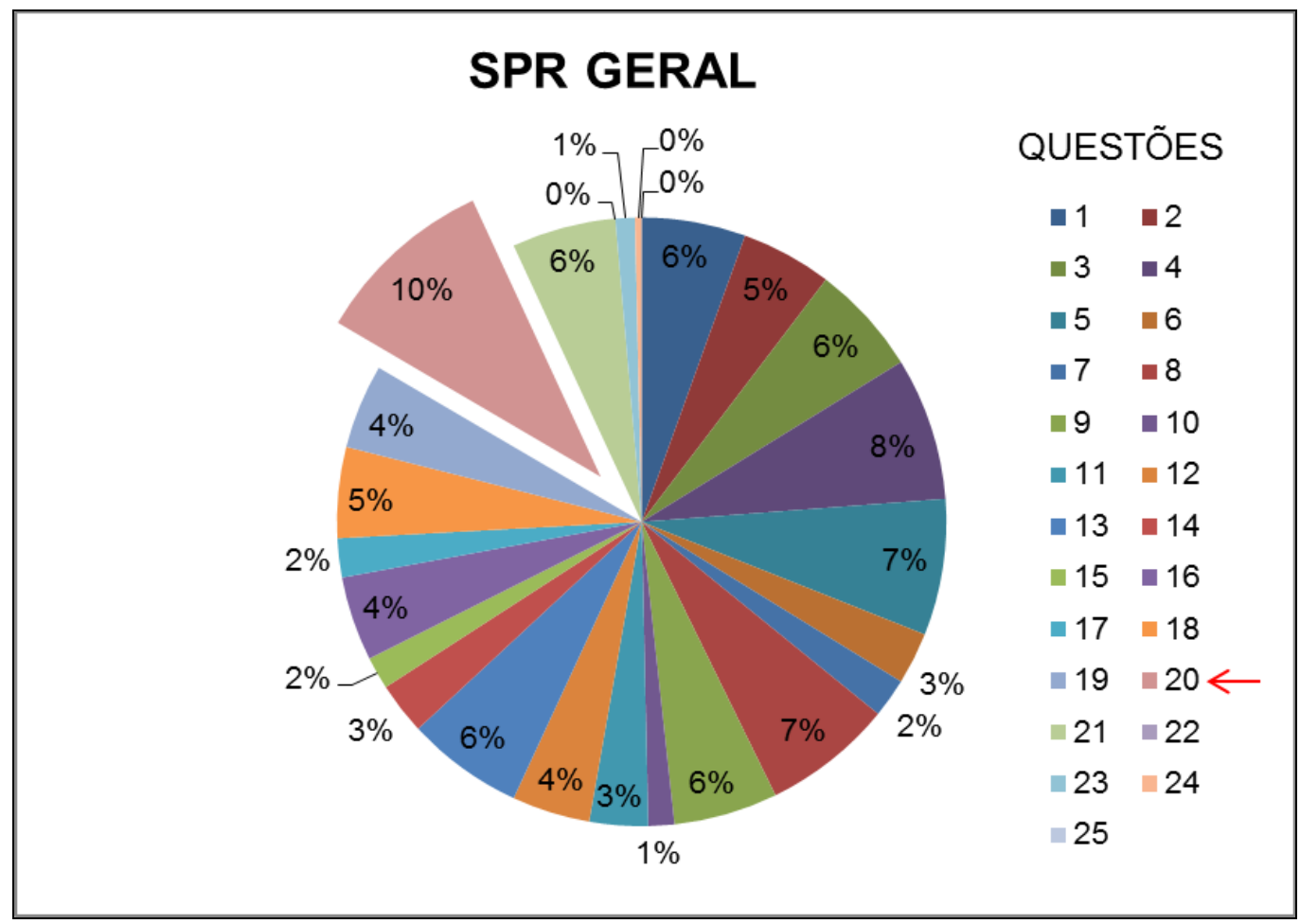

Fonte: SOUZA, 2015 
Figura 6: Percentual das mais votadas na categoria $S P R$

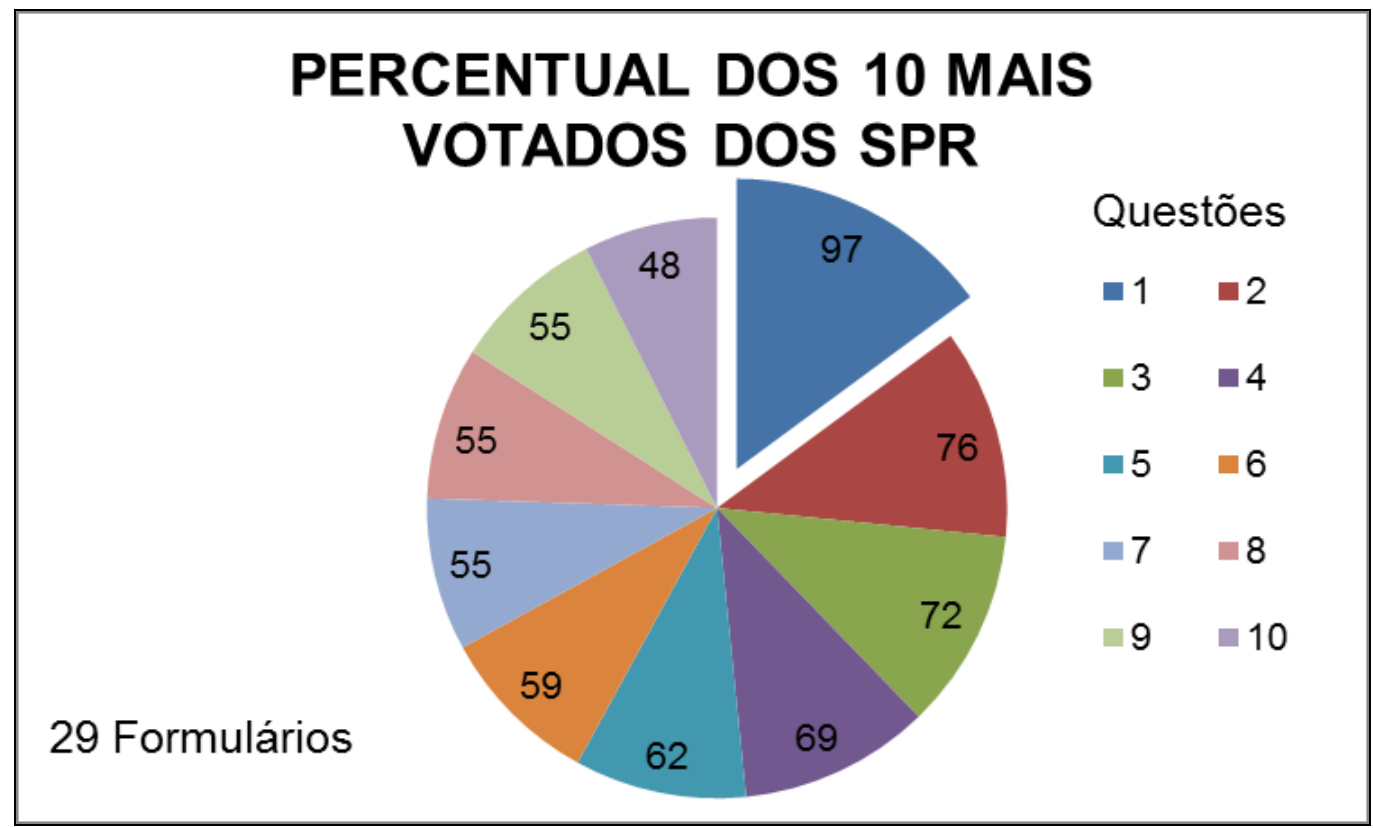

Fonte: SOUZA, 2015

Figura 7: Quantidade do número de votos da categoria SPR

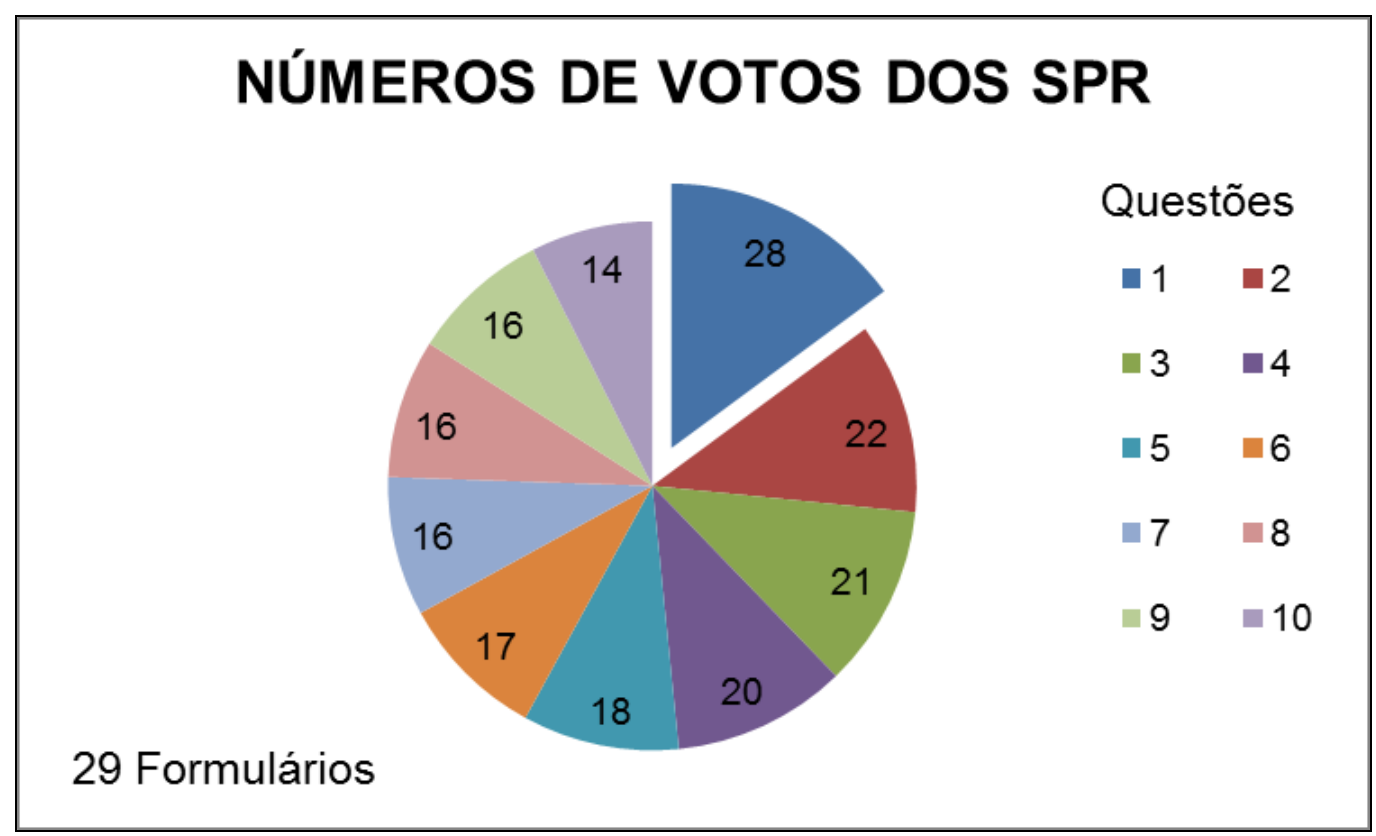

Fonte: SOUZA, 2015 
E assim, as 10 recomendações mais votadas na categoria "SPR" foram:

$1^{\text {a }}$. Use sempre um monitor individual com alarme durante todo o trabalho.

$2^{\mathrm{a}}$. Conheça muito bem como operar o tipo de irradiador a ser usado.

$3^{\mathrm{a}}$. Faça o teste do gabarito "passa-não passa" no porta-fonte e no cabo de comando.

$4^{\mathrm{a}}$. Inspecione os equipamentos de radiografia e acessórios para verificar as boas condições de uso.

$5^{\mathrm{a}}$. Monitore sempre o irradiador e o tubo-guia após recolher a fonte.

$6^{\mathrm{a}}$. Conecte corretamente os engates do cabo de comando e do porta-fonte.

$7^{\mathrm{a}}$. Avalie com antecedência o local da radiografia antes da realização do trabalho.

$8^{\text {a }}$. Mantenha o irradiador sempre vigiado.

$9^{a}$. Verifique o funcionamento correto dos monitores de radiação em uso.

10a . Comunique ao Supervisor de Radioproteção as possíveis anormalidades na operação e nos equipamentos.

Na classificação da categoria "Trabalhadores da Área" foi quantificado o resultado dos 25 participantes nas 22 recomendações, destacando-se com 8\% a questão 1 (Avalie com antecedência o local da radiografia antes da realização do trabalho) e 8 (Inspecione os equipamentos de radiografia e acessórios para verificar as boas condições de uso), sendo em $1^{\circ}$ lugar das 10 mais votadas a questão 8 com $80 \%$ (20 votos), conforme as figuras 8,9 e 10 . 
Figura 8: Resultado geral da categoria Trabalhadores da Área

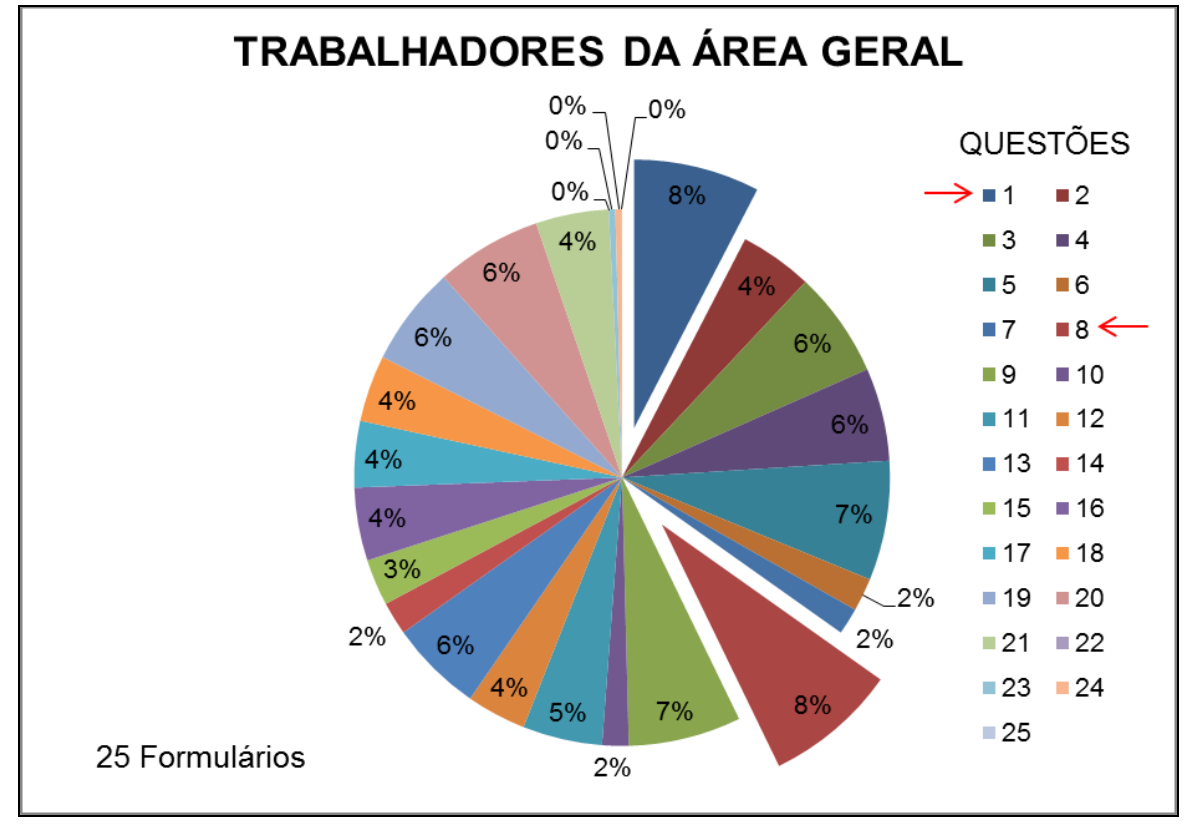

Fonte: SOUZA, 2015

Figura 9: Percentual das mais votadas na categoria Trabalhadores da Área

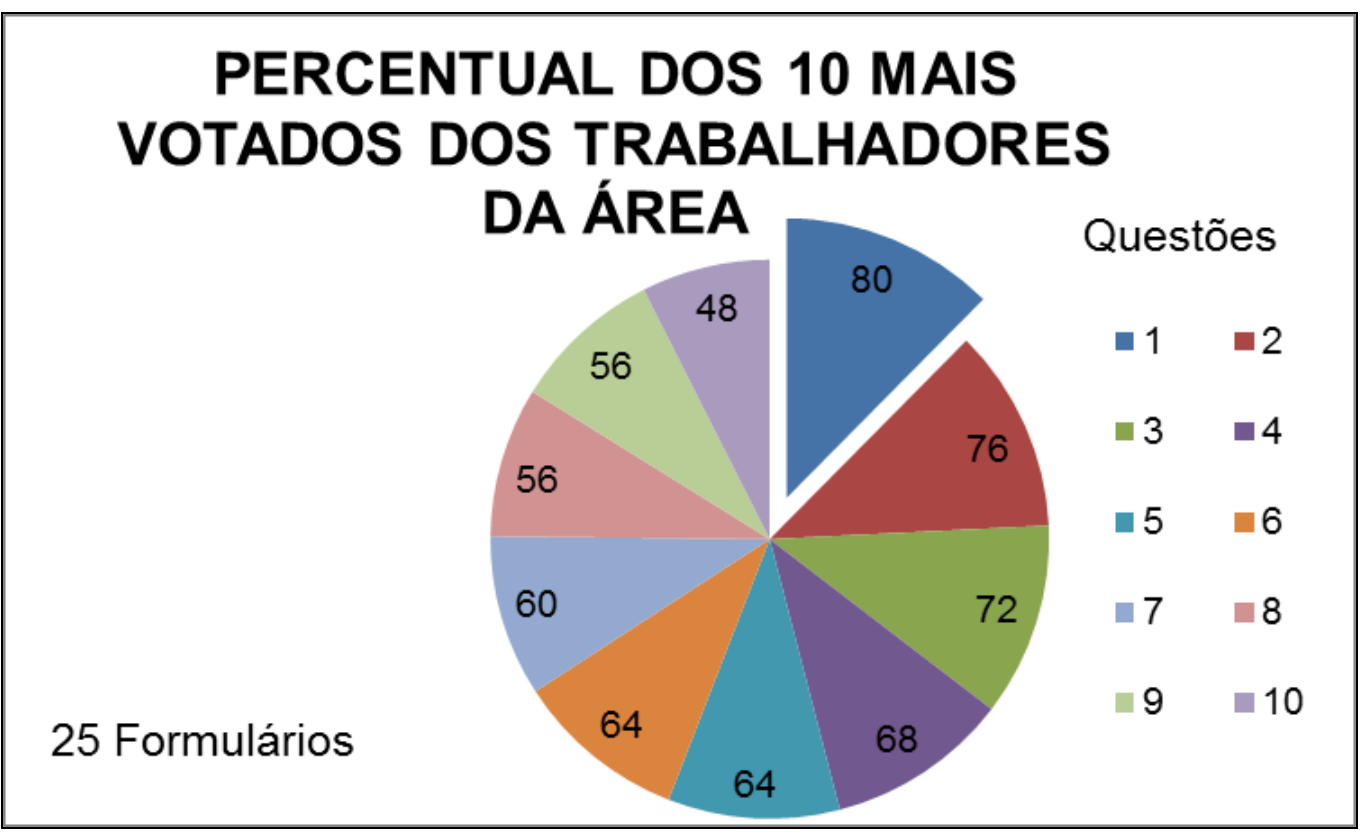

Fonte: SOUZA, 2015 
Figura 10: Quantidade do número de votos da categoria Trabalhadores da Área

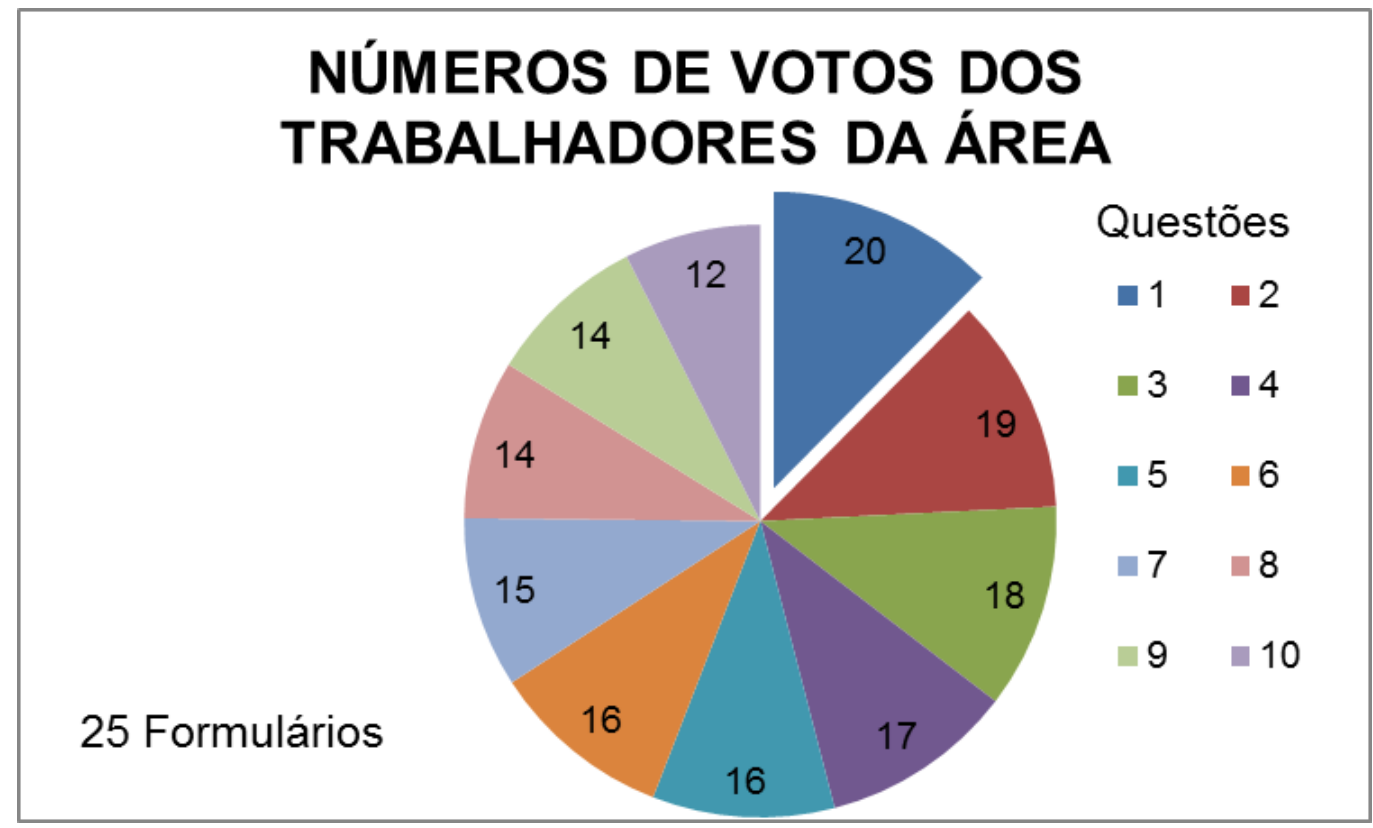

Fonte: SOUZA, 2015

E assim, as 10 mais votadas na categoria dos "Trabalhadores da Área" foram:

$1^{\text {a }}$. Inspecione os equipamentos de radiografia e acessórios para verificar as boas condições de uso.

$2^{a}$. Avalie com antecedência o local da radiografia antes da realização do trabalho.

$3^{\text {a }}$. Faça o teste com o gabarito "passa-não passa" no porta-fonte e no cabo de comando.

$4^{\mathrm{a}}$. Mantenha a área de exposição isolada e sinalizada.

$5^{\mathrm{a}}$. Use sempre um monitor individual com alarme durante todo o trabalho.

$6^{\mathrm{a}}$. Conecte corretamente os engates do cabo de comando e do porta-fonte.

$7^{\mathrm{a}}$. Use sempre o monitor de radiação ao entrar na área isolada de radiografia.

$8^{a}$. Conheça muito bem como operar o tipo de irradiador a ser usado.

$9^{\mathrm{a}}$. Monitore sempre o irradiador e o tubo-guia após recolher a fonte radioativa.

$10^{\mathrm{a}}$. Monitore a área depois de recolher a fonte radioativa ao final dos trabalhos. 
$\mathrm{Na}$ classificação da categoria "Outros (CNEN e não identificados)" foi quantificado o resultado dos 21 participantes nas 22 recomendações, destacando-se com 7\% a questão 21 (Monitore sempre o irradiador e o tubo-guia após recolher a fonte radioativa), sendo em $1^{\circ}$ lugar entre as 10 mais votadas com 76\% (16 votos), como mostram as figuras 11, 12 e 13.

Figura 11: Resultado geral da categoria Outros

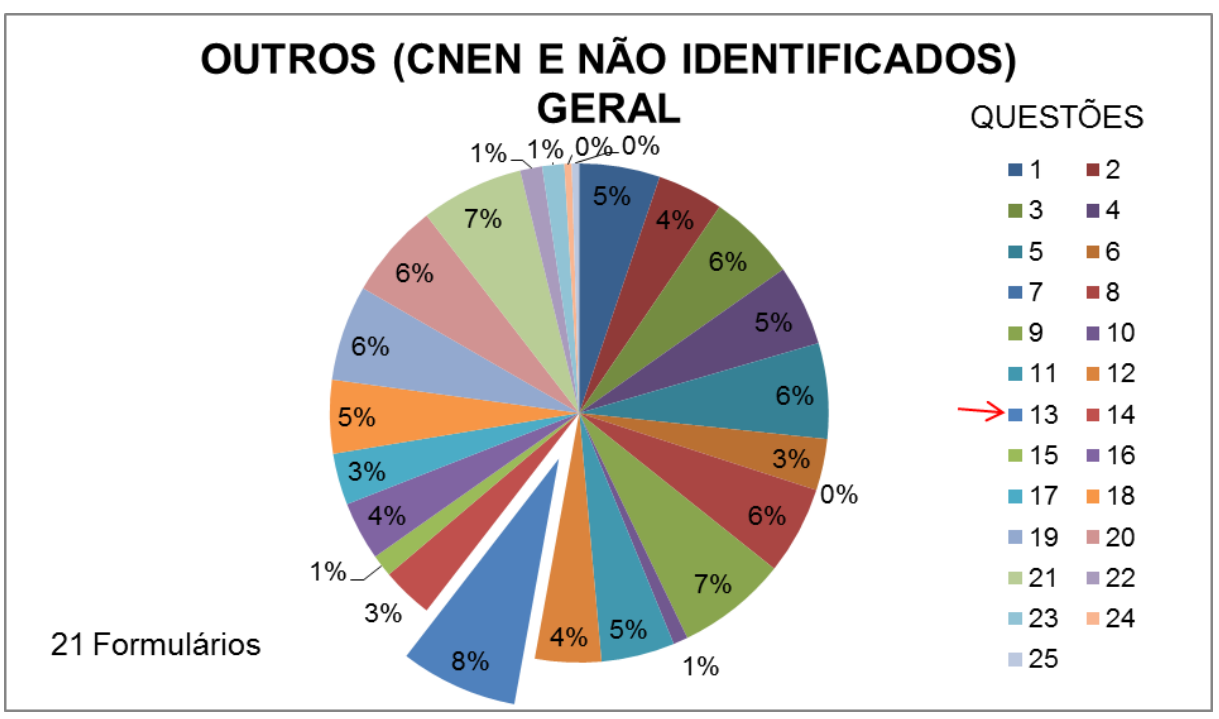

Fonte: SOUZA, 2015

Figura 12: Percentual das mais votadas na categoria Outros

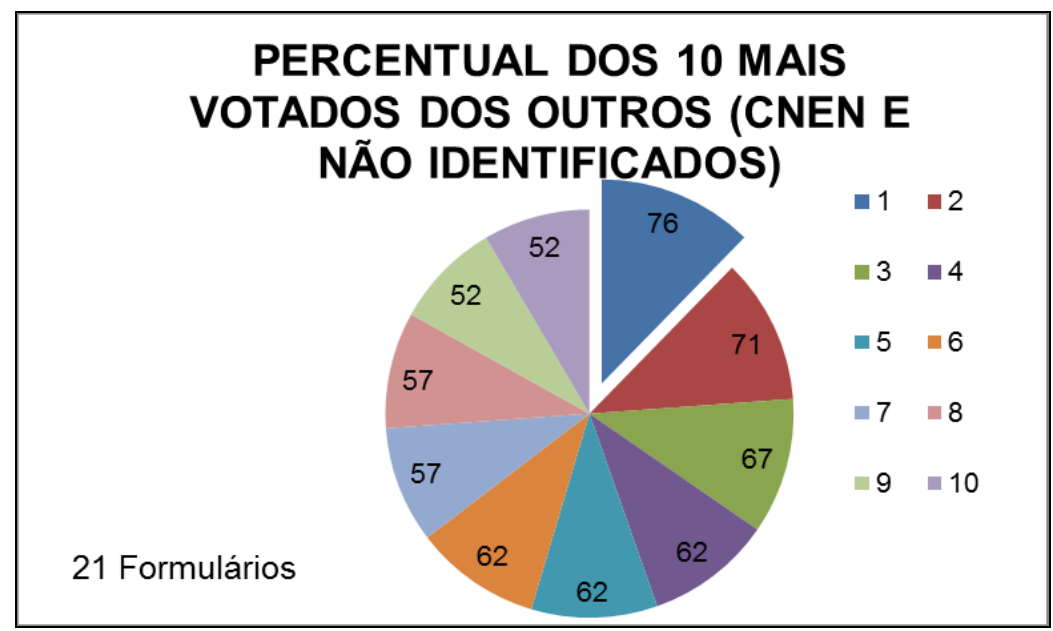

Fonte: SOUZA, 2015 
Figura 13: Quantidade do número de votos da categoria Outros

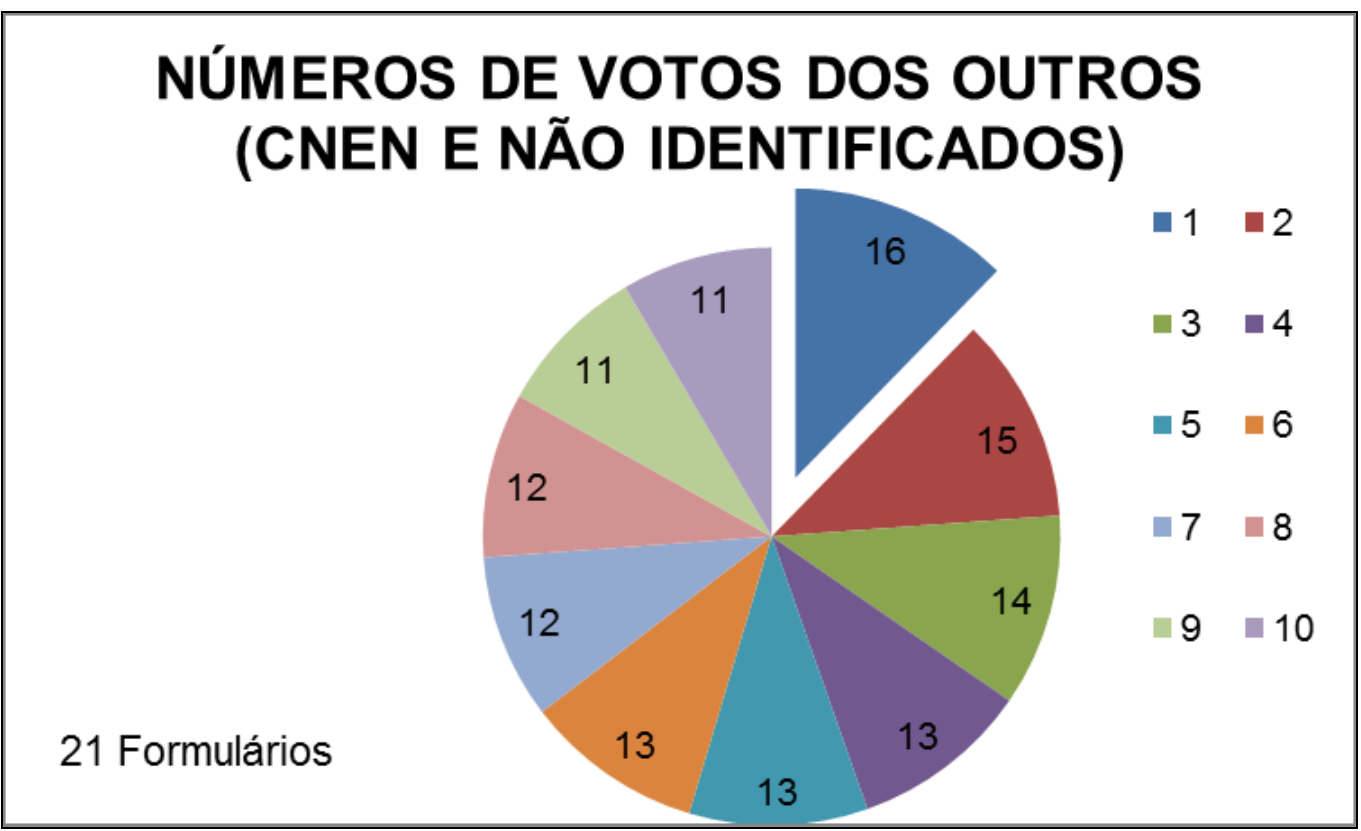

Fonte: SOUZA, 2015

E assim, as 10 mais votadas na categoria "Outros" foram:

$1^{\mathrm{a}}$. Monitore sempre o irradiador e o tubo-guia após recolher a fonte radioativa.

$2^{\mathrm{a}}$. Mantenha a área de exposição isolada e sinalizada.

$3^{\mathrm{a}}$. Verifique o funcionamento correto dos monitores de radiação em uso.

$4^{\mathrm{a}}$. Faça o teste com o gabarito "passa-não passa" no porta-fonte e no cabo de comando.

$5^{\mathrm{a}}$. Use sempre o monitor de radiação ao entrar na área isolada de radiografia.

$6^{\mathrm{a}}$. Use sempre um monitor individual com alarme durante todo o trabalho.

$7^{\text {a }}$. Conecte corretamente os engates do cabo de comando e do porta-fonte.

$8^{\mathrm{a}}$. Inspecione os equipamentos de radiografia e acessórios para verificar as boas condições de uso.

9a . Avalie com antecedência o local da radiografia antes da realização do trabalho.

$10^{\mathrm{a}}$. Conheça muito bem como operar o tipo de irradiador a ser usado. 


\subsection{Contabilização dos Votos}

De acordo com a $3^{\text {a }}$ etapa, foram contabilizados os números de votos para cada uma das 10 recomendações tomadas por base da categoria Geral, com relação às três categorias, como demonstra a figura 14 .

Figura 14: Contabilização dos votos de todas as categorias

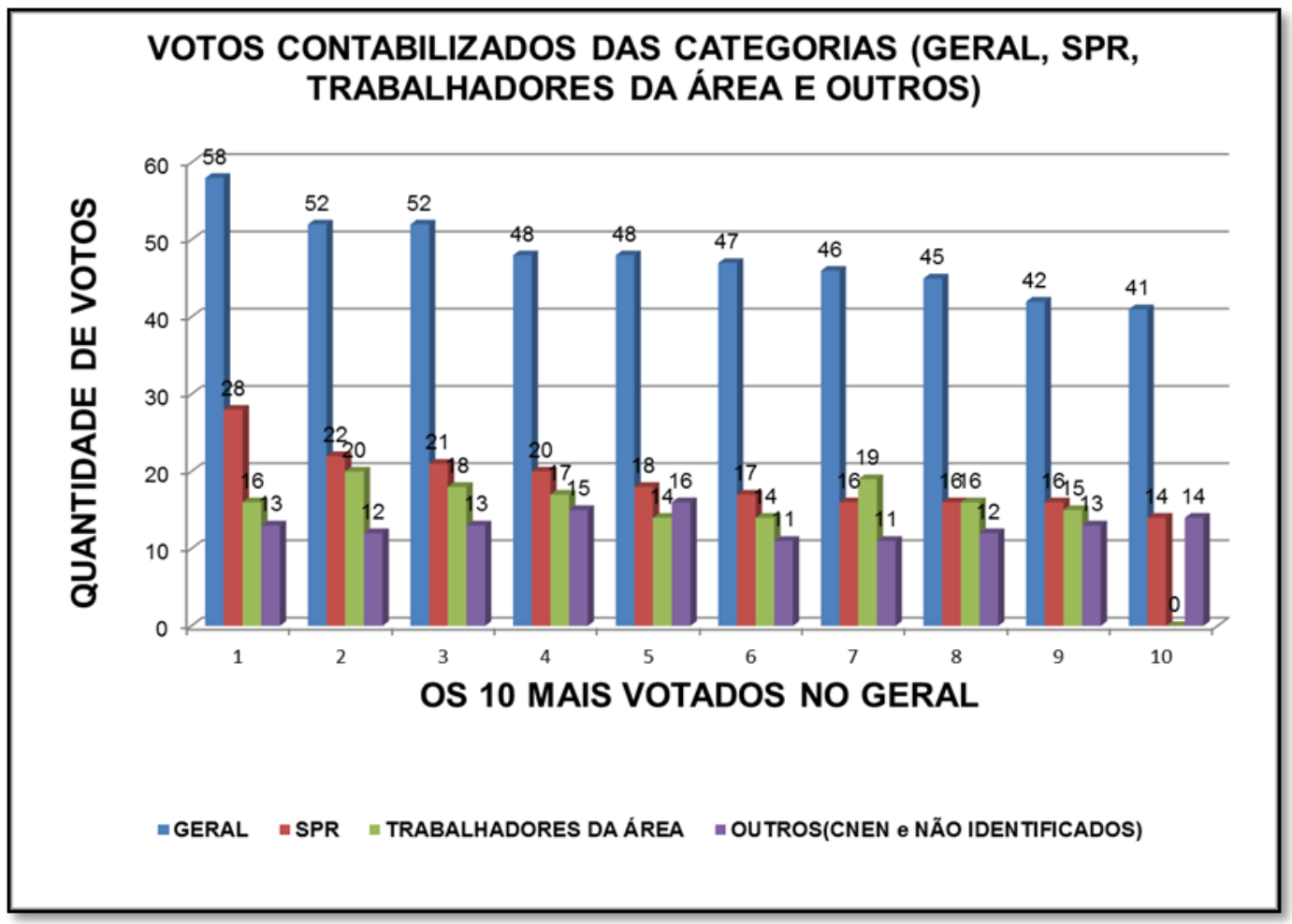

Fonte: SOUZA, 2015 


\section{CONCLUSÕES}

O objetivo principal deste trabalho foi estabelecer as 10 recomendações mais importantes para uso seguro em gamagrafia industrial, a fim de prevenir acidentes radiológicos, levando em consideração a segurança do trabalhador e também expandir os conhecimentos quanto à importância de como proceder corretamente em tal aplicação.

Conclui-se que:

1. O método estatístico "Distribuição de Frequência", escolhido mostrou-se adequado quanto aos resultados obtidos.

2. As 10 recomendações mais votados na categoria "Geral", são as mesmas das outras três categorias (SPR, Trabalhadores da área e outros), porém ordenado diferente no seu grau de importância de um (1) a dez (10).

3. Houve uma coincidência na classificação das recomendações que obtiveram o $1^{\circ}$., $3^{\circ}$., $5^{\circ}$. e $7^{\circ}$. lugar tanto para as categorias "Geral" e "SPR". A coincidência entre as categorias "Geral" e a do "Trabalhadores da área" foi somente no $3^{\mathrm{a}}$ e $10^{\mathrm{a}}$ lugar.

4. As 10 Recomendações mais Importantes para Prevenção de Acidentes Radiológicos em Gamagrafia Industrial foram às da categoria "Geral”, mostrada a seguir:

$1^{a}$. Use sempre um monitor individual com alarme durante todo o trabalho.

$2^{a}$. Inspecione os equipamentos de radiografia e acessórios para verificar as boas condições de uso.

3a. Faça o teste do gabarito "passa-não passa” no porta-fonte e no cabo de comando.

$4^{a}$. Mantenha a área de exposição isolada e sinalizada.

$5^{a}$. Monitore sempre o irradiador e o tubo-guia após recolher a fonte.

$6^{a}$. Conheça muito bem como operar o tipo de irradiador a ser usado.

$7^{a}$. Avalie com antecedência o local da radiografia antes da realização do trabalho.

$8^{a}$. Conecte corretamente os engates do cabo de comando e do porta-fonte.

$9^{a}$. Use sempre o monitor de radiação ao entrar na área isolada de radiografia.

10 . Verifique o funcionamento correto dos monitores de radiação em uso. 


\section{REFERÊNCIAS}

[1] DA SILVA, F. C. A. Uma metodologia de analise de acidentes radiológicos em gamagrafia industrial. Dissertação de Mestrado, IME, Rio de Janeiro, 1990.

IAEA - International Atomic Energy Agency. Categorization of radioactive sources. IAEATECDOC-1344. Vienna, 2003.

[2] International Atomic Energy Agency. The Radiological Accident in Nueva Aldea. IAEA Publication, Vienna, Austria, 2009.

[3] International Atomic Energy Agency. The Radiological Accident in Yanango. IAEA Publication, Vienna, Austria, 2000.

[4] VIEIRA, S. Introdução à Bioestatística. 3 ed. Rio de Janeiro, Elsevier, 1980.

[5] SOUZA, L. S. As 10 recomendações mais importantes para prevenção de acidentes radiológicos em gamagrafia industrial. Monografia Pós-Graduação, IRD, Rio de Janeiro, 2015. 